\title{
Sirolimus-associated hepatotoxicity: case report and review of the literature
}

This article was published in the following Dove Press journal:

Transplant Research and Risk Management

17 January 2012

Number of times this article has been viewed

\author{
Brock Macdonald' \\ Evi Vakiani \\ Rhonda K Yantiss ${ }^{3}$ \\ Jun Lee ${ }^{4}$ \\ Robert S Brown Jr ${ }^{5}$ \\ Samuel H Sigal ${ }^{6}$ \\ 'Division of Gastroenterology, \\ Department of Medicine, University \\ of California-San Francisco, San \\ Francisco, CA, ${ }^{2}$ Department of \\ Pathology, Memorial Sloan-Kettering \\ Cancer Center, New York, NY, \\ ${ }^{3}$ Department of Pathology and \\ Laboratory Medicine, New York \\ Weill Cornell Medical College, New \\ York, NY, ${ }^{4}$ Division of Nephrology, \\ Department of Medicine, Weill \\ Cornell Medical College, New York, \\ NY, ${ }^{5}$ Division of Gastroenterology, \\ Department of Medicine, Columbia \\ University College of Physicians and \\ Surgeons, New York, NY, ' ${ }^{6}$ ivision \\ of Gastroenterology and Hepatology, \\ Department of Medicine, New York \\ University, New York, NY, USA
}

Correspondence: Samuel H Sigal Division of Gastroenterology and Hepatology, Department of Medicine, New York University, 550 First Avenue, New York, NY 10016, USA

$\mathrm{Tel}+\mathrm{I} 2122633643$

Fax + I 212263375 |

Email samuel.sigal@nyumc.org

\begin{abstract}
The use of sirolimus as an alternative to calcineurin inhibitors for posttransplant immunosuppression is associated with a variety of inflammatory conditions. In this report, we describe the case of a 34-year-old man who developed abnormal liver tests 6 years after livedonor kidney transplantation and 5 years after initiation of sirolimus-based immunosuppression. Elevated aminotransferase levels persisted after withdrawal of potentially hepatotoxic medications, and serologic evaluation for viral hepatitis, autoimmune disease, and genetic disorders was unrevealing. Liver biopsy revealed prominent hepatocellular injury associated with a mixed inflammatory infiltrate and liver tests normalized within 2 weeks of discontinuation of sirolimus. In this report, we review previous reports of sirolimus hepatotoxicity and propose a unifying hypothesis for the various inflammatory conditions that have been attributed to sirolimus.
\end{abstract}

Keywords: sirolimus, immunosuppression, transplant, inflammatory conditions, hepatotoxicity

\section{Introduction}

Kidney transplantation is associated with significantly decreased morbidity and mortality for patients with end-stage kidney disease on hemodialysis. ${ }^{1}$ The mainstay of posttransplant immunosuppression involves the use of the calcineurin inhibitors (CNIs) cyclosporine and tacrolimus. Sirolimus (SRL) (rapamycin, Rapamune ${ }^{\text {TM}}$; Wyeth, Madison, NJ) is a macrocyclic lactone extracted from a soil sample from Easter Island and was initially developed as an antifungal agent. ${ }^{2}$ It was subsequently demonstrated to have potent immunosuppressant properties mediated by blockage of the mammalian target of rapamycin (mTOR), a protein kinase that regulates cell growth and cell cycle progression of B and T lymphocytes through signal transduction from the interleukin-2 receptor to the nucleus. Sirolimus is frequently utilized as an alternative to CNIs in cases of CNI-induced nephrotoxicity. ${ }^{3,4}$ With increasing SRL use, a variety of inflammatory extrarenal toxicities have been identified, including dermatologic, pulmonary and cardiac complications. In this report, we describe the histopathologic changes of SRL-associated hepatotoxicity and propose a unifying pathogenic mechanism for the various inflammatory conditions associated with its use.

\section{Case report}

A 34-year-old man with gout and hypertension presented with end-stage kidney disease at the age of 28 years. Kidney biopsy revealed changes consistent with chronic glomerulonephritis, and he underwent live donation kidney transplantation from his father in December 2002. Immediately following transplantation, immunosuppression submit your manuscript $\mid$ www.dovepress.com

Dovepress

http://dx.doi.org/| 0.2147/TRRM.S21313
Transplant Research and Risk Management 2012:4 I-5

(C) 2012 Macdonald et al, publisher and licensee Dove Medical Press Ltd. This is an Open Access article which permits unrestricted noncommercial use, provided the original work is properly cited. 
was achieved with tacrolimus and mycophenolate mofetil and the patient's liver function tests were noted to be normal. However, 8 months after transplantation, the mycophenolate mofetil was discontinued and SRL therapy was begun. Mildly abnormal liver tests (alanine transaminase [ALT] $50 \mathrm{U} / \mathrm{L}$ [0-45 U/L]) were first noted 26 months after initiation of SRL in September 2005, and repeat liver tests 5 months later revealed further increases (aspartate aminotransferase [AST] $150 \mathrm{U} / \mathrm{L}$ [0-45 U/L]; ALT $161 \mathrm{U} / \mathrm{L})$. Medications at that time included SRL, atorvastatin, ramipril, venlafaxine, and tacrolimus. The patient remained asymptomatic, and physical exam was unremarkable. Abdominal ultrasound 43 months after starting SRL revealed normal liver size and echogenicity, no biliary abnormalities, and patent hepatic vessels. Therapy with atorvastatin and venlafaxine were discontinued to exclude the possibility of drug-induced hepatotoxicity. Evaluations for viral hepatitis (hepatitis $C$ virus antibody, hepatitis $C$ virus RNA polymerase chain reaction (PCR), and hepatitis B virus DNA PCR) were negative. Abnormal liver tests persisted and repeated ultrasonographic imaging 51 months after starting SRL was again normal (Figure 1). One month later, a marked increase in aminotransferase levels (AST 669 U/L, ALT $868 \mathrm{U} / \mathrm{L}$ ) was noted. Medications at that time included SRL, ramipril, tacrolimus, methylphenidate, and colchicine. Serologic evaluation was unremarkable (alpha-1 anti-trypsin, $181 \mathrm{mg} / \mathrm{dL}$ [93-224 mg/dL]; ceruloplasmin, $20 \mathrm{mg} / \mathrm{dL}$ [17-54 mg/dL]; iron and iron saturation, $81 \mathrm{mg} / \mathrm{dL}$ [50-160 mg/dL] and 26\% [15\%$53 \%$ ], respectively; immunoglobulin $\mathrm{M}, 151 \mathrm{mg} / \mathrm{dL}$ [56-352 mg/dL]; immunoglobulin G, 1080 mg/dL [639-1349 $\mathrm{mg} / \mathrm{dL}])$. Antinuclear antibody; anti-smooth muscle antibody; antimitochondrial antibody; and PCR for cytomegalovirus, Epstein-Barr virus, herpes simplex virus, and varicellazoster virus were negative. Abdominal magnetic resonance imaging revealed periportal edema, no biliary dilatation, and patent hepatic vessels. Abnormal liver tests persisted after discontinuing methylphenidate and colchicine.

Percutaneous liver biopsy revealed moderate to severe active hepatitis (Figure 2). The lymphocyte-rich inflammatory infiltrate also contained easily detectable Kupffer cells, plasma cells, eosinophils, and neutrophils, and was present in both portal tracts and lobules (Figure 3). Numerous necroinflammatory foci and necrotic hepatocytes were noted within the lobules (Figure 4). Mild cholestasis in combination with focal bile duct injury was also present (Figure 5), but steatosis, granulomas, and sinusoidal dilatation were lacking. A Masson's trichrome stain demonstrated periportal and pericellular fibrosis, and moderate iron accumulation within Kupffer cells was revealed with a Prussian blue stain. SRL was discontinued with rapid and persistent normalization of AST and ALT levels within 2 weeks (Figure 1). Throughout this whole period the patient's sirolimus levels remained

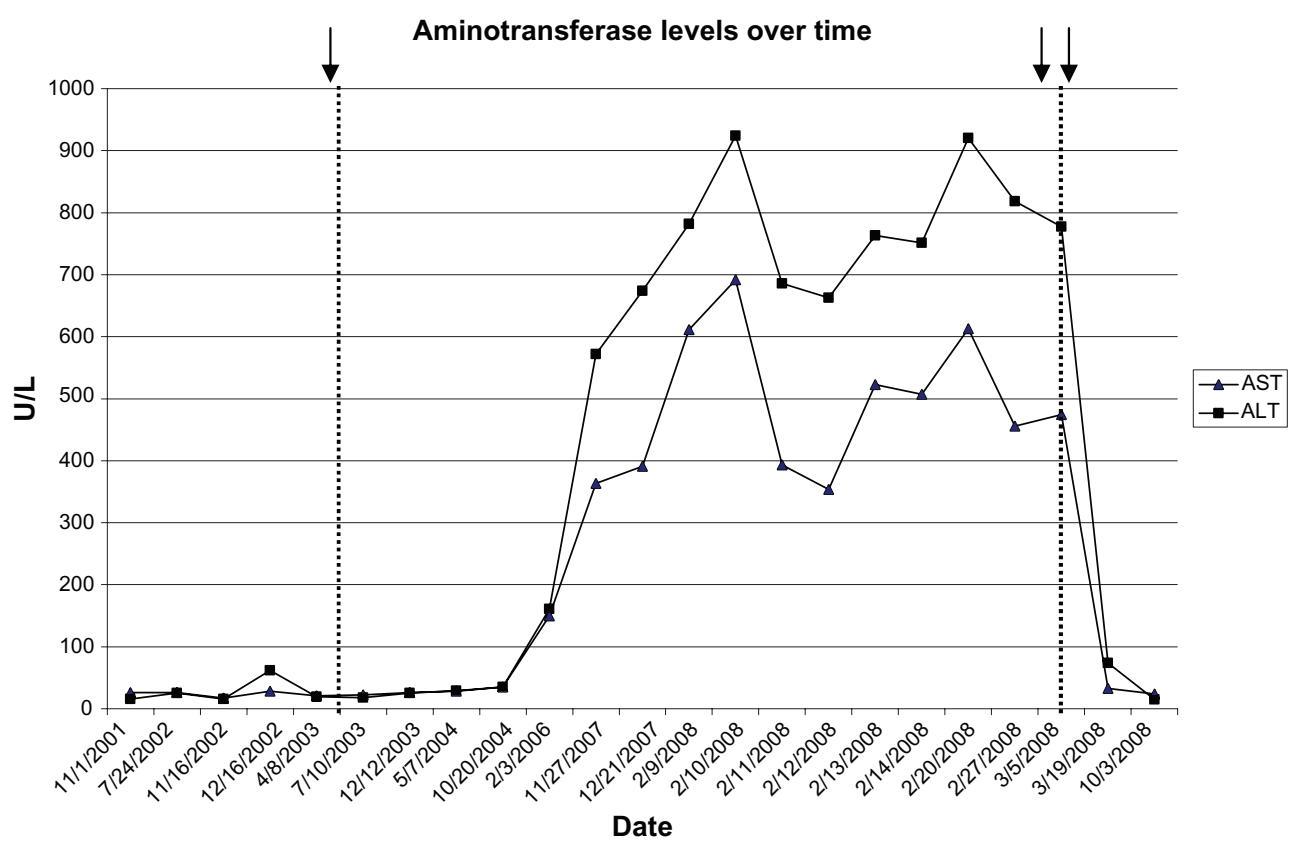

Figure I Aminotransferase levels before, during and after withdrawal of SRL immunosuppression. Except for one determination, liver tests were repeatedly normal after kidney transplantation on immunosuppression with tacrolimus and MMF. AST and ALT levels were repeatedly abnormal beginning 26 months after initiation of SRL ( $\downarrow$ ) and promptly normalized after its withdrawal $(\downarrow \downarrow)$.

Abbreviations: SRL, sirolimus; MMF, mycophenolate mofetil; AST, aspartate aminotransferase; ALT, alanine transaminase. 


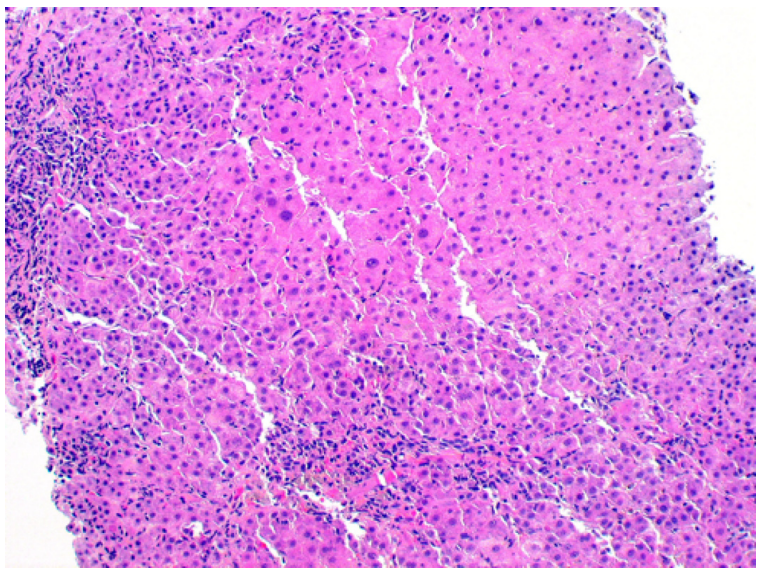

Figure $\mathbf{2}$ Liver biopsy revealed a dense inflammatory infiltrate in the portal tracts and lobules (hematoxylin and eosin stain, 100× magnification).

within the normal therapeutic range. His creatinine remained stable within normal limits.

\section{Discussion}

Hepatotoxicity is a potential but underrecognized complication of SRL immunosuppression in transplant recipients..$^{3-7}$ Although hepatotoxicity is not listed as an indication for discontinuation of therapy, initial studies indicated that $5 \%-8 \%$ of renal allograft recipients developed elevated aminotransferase levels within the first 11 months of its administration. ${ }^{6}$ Among liver transplant recipients, SRL has been reported to be associated with a similar incidence of hepatotoxicity. In a single-center evaluation of 210 patients who received SRL-based immunosuppression, ten patients (4.7\%) developed hepatotoxicity. In these cases, the initial rise in aminotransferase levels was attributed to rejection but increased SRL doses led to a further rise in levels. In liver

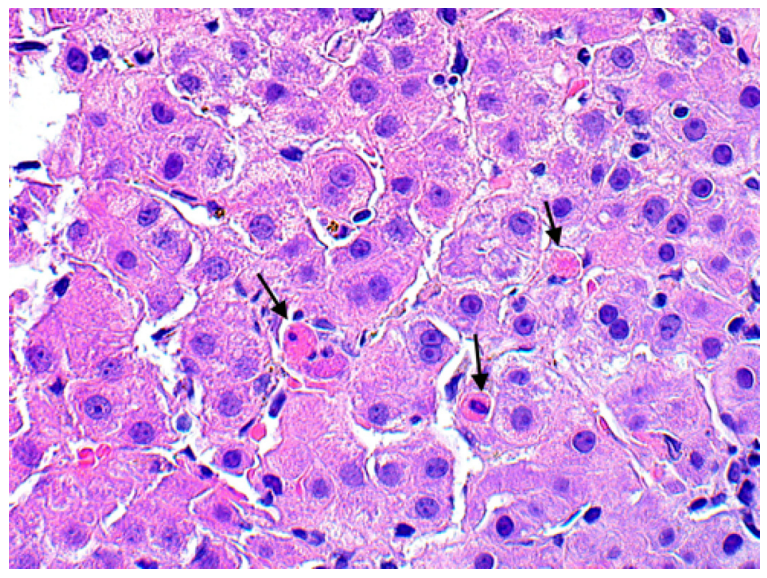

Figure 3 The inflammatory infiltrate consisted of lymphocytes, Kupffer cells, plasma cells, and granulocytes, and eosinophils were readily detected (hematoxylin and eosin stain, $400 \times$ magnification).

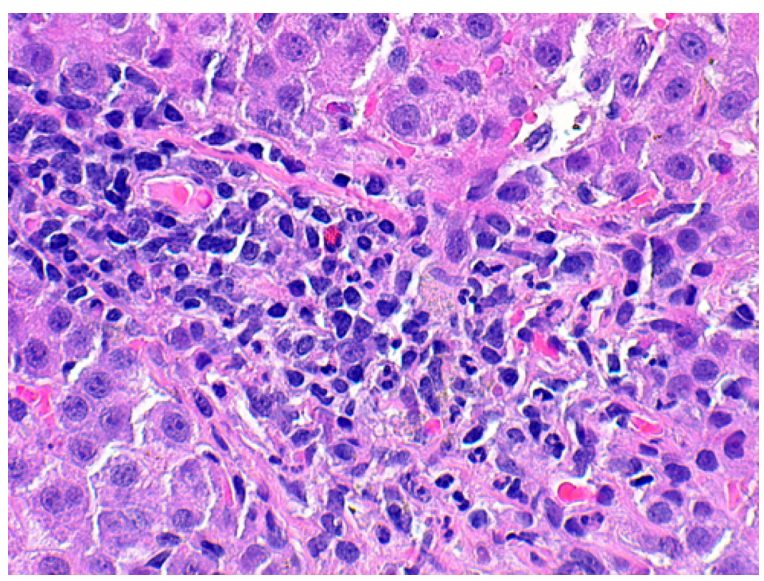

Figure 4 Scattered necroinflammatory foci and apoptotic hepatocytes were present in the lobules (hematoxylin and eosin stain, $400 \times$ magnification).

allograft recipients, abnormal liver tests appeared to occur more rapidly than in kidney transplant patients at a mean of 21 days (range 7-40), suggesting either greater sensitivity of the liver allograft to the possible toxic effects of SRL or an increased vigilance of hepatologists in monitoring liver enzymes. In a subsequent investigation evaluating SRLassociated hepatotoxicity in renal transplant recipients, hepatotoxicity developed in $25 \%$ of patients with hepatitis B or C as compared with $8.3 \%$ without viral hepatitis. ${ }^{3}$ Although a statistical difference was not present, the study was limited by the inclusion of relatively few patients. In both the kidney and liver transplant recipients, however, prompt normalization of liver tests usually occurred within 3-4 weeks of discontinuation of SRL.,3,7

Reports of the histopathology of SRL hepatotoxicity are limited to a case report of a 30-year-old kidney transplant recipient who developed abnormal liver tests 16 months

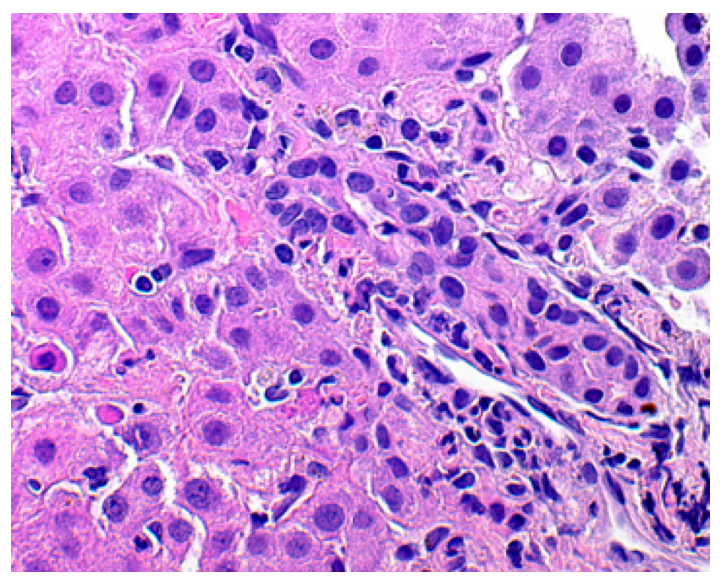

Figure 5 Although not striking, some of the bile ducts displayed regenerative epithelial cell changes in combination with infiltration by inflammatory cells (hematoxylin and eosin stain, $400 \times$ magnification). 
after starting SRL and six cases in a series of liver transplant recipients. ${ }^{3,7}$ The liver biopsy findings of the renal transplant recipient included mild expansion of the portal tracts by lymphocytes with mild interface hepatitis and focal lobular inflammation. ${ }^{7}$ Liver biopsies performed in the six liver transplant recipients showed grade 2 inflammation in only one case. ${ }^{4}$ Histologic changes consistent with sinusoidal congestion were observed in two patients, while one biopsy showed eosinophilia. ${ }^{4}$

The hepatitis in our case can be attributed to SRLassociated hepatotoxicity due to the exclusion of other causes, onset after initiation of SRL therapy, and prompt resolution after its withdrawal. Other potential hepatotoxic agents were withdrawn without improvement in liver tests, and other causes of liver disease including viral, metabolic, and genetic were excluded. For definite determination of the role SRL as the offending agent, rechallenge with SRL would have been required. However, this was deemed not to be appropriate given the presumptive diagnosis with prompt normalization aminotransferase levels after withdrawal and the ready availability of an alternative immunosuppressive agent.

Our case represents the most complete report of the histopathology of SRL-associated hepatotoxicity to date. In the previous case reports, histopathological details were scant., ${ }^{3,47}$ In the series of the liver transplant recipients with abnormal liver tests on SRL, potential concomitant processes of rejection and/or viral hepatitis prevent an absolute determination of the contribution of SRL to the histopathology.

The liver biopsy in our patient showed significantly higher inflammation and hepatocellular damage compared to previous reported cases of SRL-associated hepatotoxicity, mirroring the higher elevation in aminotransferase levels seen in our case. ${ }^{3,4,7}$ These findings could be due to the prolonged duration of SRL use after the onset of hepatotoxicity. Similarly, it was noted that increased doses of SRL resulted in higher aminotransferase levels in liver transplant recipients, suggesting a possible dose-dependent relationship.

Although the exact mechanism of SRL-induced injury remains to be determined, the presence of plasma cells and eosinophils suggest an underlying immune-mediated process. In support of this proposal, the pathology of SRLhepatotoxicity is similar to that of other forms of SRL-induced inflammatory toxicities, including leukocytoclastic vasculitis, interstitial pneumonitis, bronchiolitis obliterans organizing pneumonia, diffuse alveolar hemorrhage, pericarditis, and mucositis. ${ }^{8-13}$ In addition, the clinical response of SRLinduced oral aphthous ulcers to the topical application of the potent steroid clobetasol suggests a similar immune-mediated pathologic process. ${ }^{8}$ Mechanisms that have been proposed for SRL-induced immune-mediated pulmonary toxicity include SRL-associated exposure of cryptic alveolar antigens and hapten formation between SRL and high-affinity plasma proteins that induces Th- 1 activation and cytokine release. ${ }^{14,15}$ The inflammatory infiltrate present in our case suggests a possible similar mechanism of immune activation within the liver.

The paucity of significant fibrosis in our case is a striking finding given the prolonged injury of over 2 years with significant inflammation and marked aminotransferase elevation. Clinically, this effect is associated with impaired wound healing in the early posttransplant period. ${ }^{16-18}$ SRL has been shown to inhibit wound healing, possibly by the inhibition of platelet-derived growth factor, which activates fibroblasts. As well, there appears to be decreased angiogenesis due to a decrease of endothelial expressed vascular endothelial growth factor receptors. ${ }^{16}$ In the liver, SRL inhibits de novo fibrinogenesis by decreasing the activity of stellate cells, which are the primary source of fibrin deposition..$^{16,17}$ Although speculative, we propose that these unique properties account for the limited fibrosis in both SRL-associated hepatic and pulmonary toxicity. ${ }^{15,18}$ In addition, activation of mTOR receptor by SRL, which inhibits autophagy, may play a further role in the pathogenesis of SRL-associated drug toxicity and contribute to the especially prominent number of apoptic bodies observed in our case.

SRL is an important addition to the armamentarium of posttransplant immunosuppression and is frequently employed for prevention of calcineurin-associated nephrotoxicity. ${ }^{5}$ With the widespread use of SRL, however, an increasing number of potential toxicities have been identified. Our case emphasizes the need to include hepatotoxicity in the differential diagnosis of abnormal liver tests in the posttransplant patient on SRL. An increased awareness of the potential for SRL-associated hepatotoxicity is particularly important in the management of liver transplant recipients with chronic hepatitis in whom abnormal liver tests are common.

\section{Disclosure}

The authors report no conflicts of interest in this work.

\section{References}

1. Straathof-Galema L, van Saase JL, Verburgh CA, et al. Morbidity and mortality during renal replacement therapy: dialysis versus transplantation. Clin Nephrol. 2001;55(3):227-232.

2. Sehgal SN, Baker H, Vezina C. Rapamycin (AY-22,989), a new antifungal antibiotic. II. Fermentation, isolation and characterization. J Antibiot (Tokyo). 1975;28(10):727-732. 
3. Chang HR, Lin CC, Lian JD. Lack of hepatotoxicity upon sirolimus addition to a calcineurin inhibitor-based regimen in hepatitis virus positive renal transplant recipients. Transplant Proc. 2007;39(5):1520-1522.

4. NeffGW, Ruiz P, Madariaga JR, et al. Sirolimus-associated hepatotoxicity in liver transplantation. Ann Pharmacother. 2004;38(10):1593-1596.

5. Gürkan A, Kaçar S, Erdoğdu U, et al. The effect of sirolimus in the development of chronic allograft nephropathy. Transplant Proc. 2008; 40(1):114-116.

6. Kreis H, Cisterne JM, Land W, et al. Sirolimus in association with mycophenolate mofetil induction for the prevention of acute graft rejection in renal allograft recipients. Transplantation. 2000;69(7):1252-1260.

7. Niemczyk M, Wyzgał J, Perkowska A, Porowski D, Paczek L. Sirolimus-associated hepatotoxicity in the kidney graft recipient. Transplant International. 2005;18(11):1302-1303.

8. Chuang P, Langone AJ. Clobetasol ameliorates ulceration in renal transplant patient on sirolimus. Am J Transplant. 2007;7(3):714-717.

9. Garrean S, Massad MG, Tshibaka M, Hanhan Z, Caines AE, Benedetti E. Sirolimus-associated interstitial pneumonitis in solid organ transplant recipients. Clin Transplant. 2005;19(5):698-703.

10. Hardinger KL, Cornelius LA, Trulock EP 3rd, Brennan DC. Sirolimus-induced leukocytoclastic vasculitis. Transplantation. 2002; 74(5):739-743.
11. Mahalati K, Murphy DM. Bronchiolitis Obliterans and organizing pneumonia in renal transplant recipients. Transplantation. 2000;69:1531.

12. Morath C, Schwenger V, Ksoll-Rudek D, et al. Four cases of sirolimus-associated intersitial pneumonitis: identification of risk factors. Transplant Proc. 2007;39(1):99-102.

13. Vlahakis NE, Rickman OB, Morgenthaler T. Sirolimus-associated diffuse alveolar hemorrhage. Mayo Clin Proc. 2004;79(4):541-545.

14. Morelon E, Stern M, Israël-Biet D, et al. Characteristics of sirolimus-associated intersitial pneumonitis in renal transplant patients. Transplantation. 2001;72(5):787-790.

15. Ekici Y, Emiroglu R, Ozdemir H, et al. Effect of rapamycin on wound healing: an experimental study. Transplant Proc. 2007;39(4): 1201-1203.

16. Pham PT, Pham PC, Danovitch GM, et al. Sirolimus-associated pulmonary toxicity. Transplantation. 2004;77(8):1215-1220.

17. Neef M, Ledermann M, Saegesser H, Schneider V, Reichen J. Low-dose oral rapamycin treatment reduces fibrogenesis, improves liver function, and prolongs survival in rats with established liver cirrhosis. J Hepatol. 2006;45(6):786-796.

18. Valente JF, Hricik D, Weigel K, et al. Comparison of sirolimus vs mycophenolate mofetil on surgical complications and wound healing in adult kidney transplantation. Am J Transplant. 2003;3(9):1128-1134.
Transplant Research and Risk Management

\section{Publish your work in this journal}

Transplant Research and Risk Management is an international, peerreviewed open access journal focusing on all aspects of transplantation and risk management to achieve optimal outcomes in the recipient improving survival and quality of life. The journal welcomes submitted papers covering original research, basic science, clinical studies,

\section{Dovepress}

reviews \& evaluations, guidelines, expert opinion and commentary, case reports and extended reports. The manuscript management system is completely online and includes a very quick and fair peer-review system, which is all easy to use. Visit http://www.dovepress.com/ testimonials.php to read real quotes from published authors. 